\title{
Microlensing variability in time-delay quasars ${ }^{\star}$
}

\author{
D. Paraficz ${ }^{1,2}$, J. Hjorth ${ }^{1}$, I. Burud ${ }^{3}$, P. Jakobsson ${ }^{1}$, and Á. Elíasdóttir ${ }^{1}$ \\ ${ }^{1}$ Dark Cosmology Centre, Niels Bohr Institute, University of Copenhagen, Juliane Maries Vej 30, 2100 Copenhagen, Denmark \\ e-mail: danutas@astro.ku.dk \\ 2 Nordic Optical Telescope (NOT), Apartado 474, 38700 Santa Cruz de La Palma, Canary Islands, Spain \\ 3 Norwegian Meteorological Institute, PO Box 43, Blindern, 0313 Oslo, Norway
}

Received 26 April 2006 / Accepted 15 May 2006

\begin{abstract}
We have searched for microlensing variability in the light curves of five gravitationally lensed quasars with well-determined time delays: SBS 1520+530, FBQ 0951+2635, RX J0911+0551, B1600+434 and HE 2149-2745. By comparing the light curve of the leading image with a suitably time offset light curve of a trailing image we find that two (SBS 1520+530 and FBQ 0951+2635) out of the five quasars have significant long-term ( years) and short-term $(\sim 100$ days $)$ brightness variations that may be attributed to microlensing. The short-term variations may be due to nanolenses $10^{-4}-10^{-3} M_{\odot}$, relativistic hot or cold spots in the quasar accretion disks, or coherent microlensing at large optical depth.
\end{abstract}

Key words. gravitational lensing

\section{Introduction}

The effect of a background source being gravitationally lensed by foreground compact objects is known as microlensing. Chang \& Refsdal (1979) predicted that in lensed quasar systems the light path should be affected by stars in the lensing galaxy. Moving compact objects in the lensing galaxy can cause spectral changes, brightness variability and, in the case of multiple images, flux ratio anomalies in the lensed quasar. When the foreground galaxy causes multiple images of the quasar, photometric monitoring can be used to isolate intrinsic quasar variability from microlensing variability by comparison of the separate light curves. Likewise, spectral differences between quasar images, caused by differential magnification across the quasar, can be directly detected in multiply imaged quasars. Microlensing offers the opportunity to study the nature of matter in foreground galaxies and the spatial structure of the lensed quasar at very high angular resolution (Kochanek 2004). The first quasar microlensing events were discovered 17 years ago by Irwin et al. (1989) and Schild (1990).

In this Letter we analyze the light curves of five lensed quasars for which a time delay has previously been measured in dedicated monitoring campaigns (Burud et al. 2000, 2002a,b; Hjorth et al. 2002; Jakobsson et al. 2005). Four of them were observed at the Nordic Optical Telescope (NOT) between 1998-2002 and one (HE 2149-2745) was observed at the Danish $1.5-\mathrm{m}$ telescope. We here examine the light curves for microlensing variability and at the same time make all data points available online at the CDS.

* Tables 3-8 are only available in electronic form at the CDS via anonymous ftp to cdsarc.u-strasbg.fr $(130.79 .128 .5)$ or via http://cdsweb.u-strasbg.fr/cgi-bin/qcat?J/A+A/455/L1

\section{Analysis}

The images of a lensed quasar may vary due to intrinsic quasar brightness changes and/or microlensing. Microlensing affects the light paths of each image differently (in the simplest case only one path is affected) whereas the intrinsic variations show up in all the images but at different times due to the time delay. Therefore, one can isolate the microlensing signal by calculating the difference between two light curves, suitably shifted in time to correct for the time delay.

Observationally, quasar light curves suffer from sampling effects leading to a need for interpolation. Due to their small separations and the presence of the lensing galaxy they may also potentially be affected by systematic errors in the photometry. To eliminate artifacts arising from such effects we do not interpolate data points with a gap bigger then a time delay of a system and we require that the light curve difference be (1) uncorrelated with the quasar variations and (2) independent of which image light curve we interpolate. These are conservative criteria for detection of a microlensing signal in the light curves which ensure that the signal is reliable and robust. If the criteria are violated the variability may be the result of observational, systematic or interpolation errors.

\section{Results}

Were we apply the analysis to the five quasar light curves to search for microlensing events.

\subsection{SBS $1520+530$}

The doubly imaged quasar SBS $1520+530$ (see Table 1) was monitored between February 1999 and May 2001 at the NOT. In Fig. 1 we show the light curves of the two images, A and $\mathrm{B}$, where $\mathrm{B}$ has been shifted in both time ( -130 days) and 
Table 1. Properties of the lens systems. The time delays were obtained by: 1. Burud et al. (2002b), 2. Jakobsson et al. (2005), 3. Burud et al. (2002a), 4. Hjorth et al. (2002), 5. Burud et al. (2000).

\begin{tabular}{llllll}
\hline \hline Name & $z_{1}$ & $z_{\mathrm{s}}$ & $\Delta t$ (days) & Separation & Ref. \\
\hline SBS 1520+530 & 0.72 & 1.86 & $130 \pm 2$ & $1.57^{\prime \prime}$ & 1 \\
FBQ 0951+2635 & 0.24 & 1.25 & $16 \pm 2$ & $1.10^{\prime \prime}$ & 2 \\
HE 2149-2745 & 0.495 & 2.03 & $103 \pm 12$ & $1.70^{\prime \prime}$ & 3 \\
RX J0911+0551 & 0.77 & 2.80 & $146 \pm 4$ & $3.10^{\prime \prime}$ & 4 \\
B1600+434 & 0.41 & 1.59 & $51 \pm 2$ & $1.38^{\prime \prime}$ & 5 \\
\hline
\end{tabular}

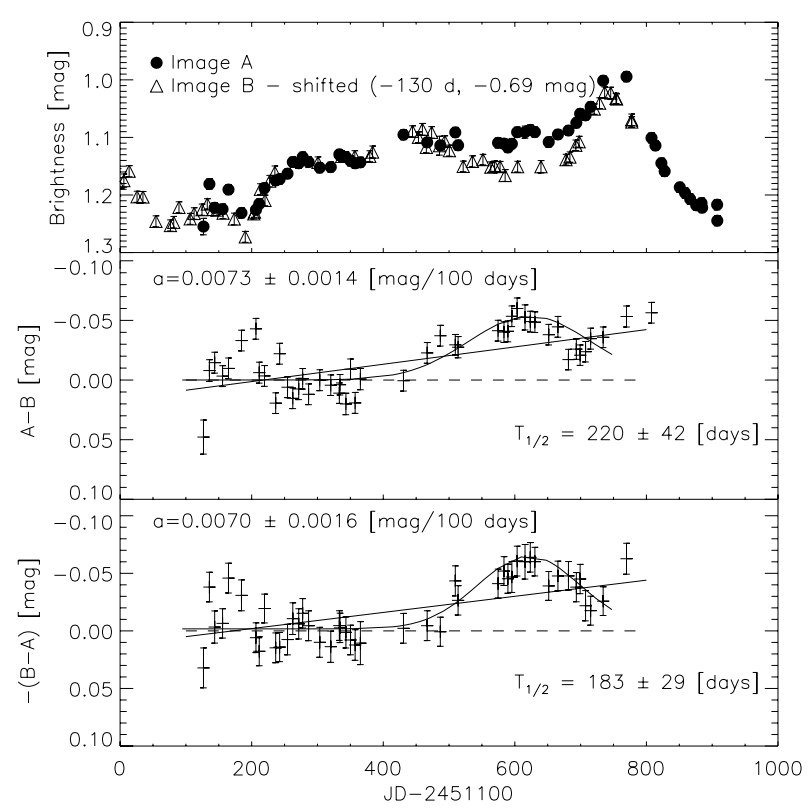

Fig. 1. $R$-band light curves of SBS $1520+530$. Top: time-delay shifted light curves, with the $\mathrm{B}$ image offset by -0.69 mag and -130 days. Middle: difference between linearly interpolated A image and B image. Bottom: difference between linearly interpolated B image and A image. The middle and bottom panels also present linear fits (where $a$ is the slope) and Gaussian fits (where $T_{1 / 2}$ is the $F W H M$ ) to the data.

brightness (-0.69 mag). Also shown are the magnitude residuals, $\Delta m$, obtained by linear interpolation of one of the images. The middle panel shows the difference between a linearly interpolated $\mathrm{A}$ light curve and the $\mathrm{B}$ data points $\left(\Delta m_{\mathrm{AB}}\right)$. The lower panel shows the difference between a linearly interpolated $\mathrm{B}$ light curve and the $\mathrm{A}$ data points $\left(\Delta m_{\mathrm{BA}}\right)$. Any deviation from a constant difference lightcurve (dashed horizontal line) can be attributed to microclensing. In both residual plots one notices a constant magnitude increase and an approximately 200-daywide magnitude bump. In the following we characterize such residuals phenomenologically using linear or Gaussian fits.

A linear fit shows that the slopes of the plots are $0.0073 \pm 0.0014 \mathrm{mag} / 100$ days for $\Delta m_{\mathrm{AB}}$ and $0.0070 \pm$ $0.0016 \mathrm{mag} / 100$ days for $\Delta m_{\mathrm{BA}}$. The agreement between these values indicates that the mean difference between the images increases by about 0.007 mag per 100 days. This is consistent with the observations of Gaynullina et al. (2005) who found an increase in the magnitude difference between the quasar images of $0.14 \pm 0.03$ mag over 1500 days $(0.009 \pm 0.002 \mathrm{mag} / 100$ days $)$. We interpret this signal as evidence for microlensing.

A Gauss function with baseline zero, fitted to the bump for $\Delta m_{\mathrm{AB}}$ gives a $F W H M$ of $220 \pm 42$ days and a peak magnitude variation of $0.053 \pm 0.008 \mathrm{mag}$. For $\triangle m_{\mathrm{BA}}$ the $F W H M$ is $183 \pm$ 29 days and the peak magnitude variation is $0.063 \pm 0.009$ mag. These values are consistent within the errors.

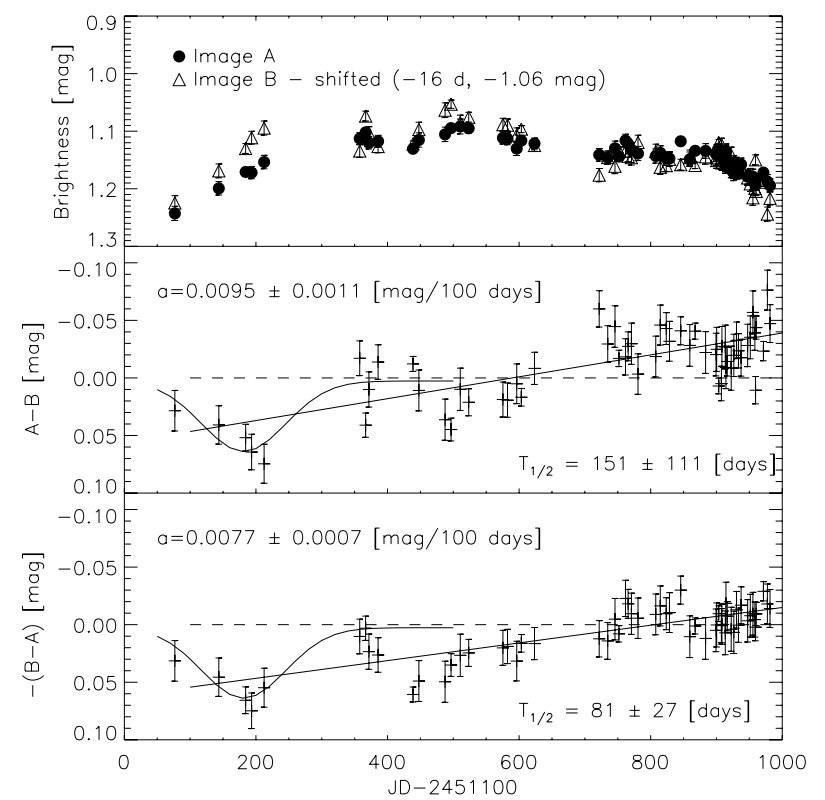

Fig. 2. $R$-band light curves of FBQ 0951+2635. Top: time-delay shifted light curves, with the $\mathrm{B}$ image offset by -1.06 mag and -16 days. Middle: difference between linearly interpolated A image and B image. Bottom: difference between linearly interpolated B image and A image. The middle and bottom panels also present linear fits (where $a$ is the slope) and Gaussian fits (where $T_{1 / 2}$ is the $F W H M$ ) to the data.

Microlensing in SBS $1520+530$ was already detected spectroscopically by Burud et al. (2002b) who analyzed continuum normalized spectra and showed that the equivalent widths of emission lines in A were larger than in B.

\section{2. $F B Q 0951+2635$}

The doubly imaged quasar FBQ 0951+2635 (see Table 1) was observed between March 1999 and June 2001 at the NOT. Spectroscopic indications of possible microlensing in the system were found by Schechter et al. (1998) and Jakobsson et al. (2005).

We repeat the microlensing-extraction procedure described above for FBQ 0951+2635. In Fig. 2 we show the light curves of the two images, $A$ and $B$, the latter shifted in time by -16 days and in brightness by $-1.06 \mathrm{mag}$. The middle and lower panels show $\Delta m_{\mathrm{AB}}$ and $\Delta m_{\mathrm{BA}}$. In both plots one notices a constant magnitude increase and a bump at the beginning of the observations.

Linear fits to the data yield slopes of $0.0095 \pm 0.0011$ and $0.0077 \pm 0.0007 \mathrm{mag} / 100$ days for $\Delta m_{\mathrm{AB}}$ and $\Delta m_{\mathrm{BA}}$ respectively. A Gauss function with baseline zero, fitted to the bump for $\triangle m_{\mathrm{AB}}$ gives a $F W H M$ of $151 \pm 111$ days and a peak magnitude variation of $0.048 \pm 0.009$ mag. For $\Delta m_{\mathrm{BA}}$ gives $F W H M$ of $81 \pm$ 27 days and a peak magnitude variation of $0.061 \pm 0.016$ mag. These values are consistent within the errors.

\subsection{Quasars where no microlensing is confirmed}

The three quasars where no microlensing variability was reliably detected are the double quasar HE 2149-2745 observed at the Danish 1.5-m telescope, ESO-La Silla (October 1998-December 2000), the quadruple quasar RX J0911+0551 observed at the NOT (March 1997April 2001), and the doubly imaged quasar B1600+434 


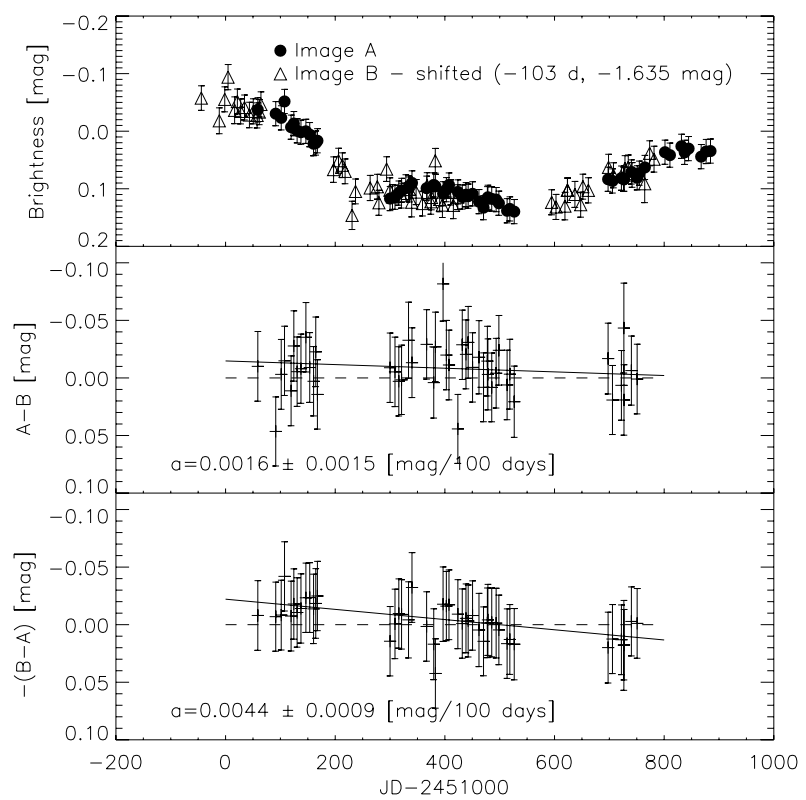

Fig. 3. $V$-band light curves of HE 2149-2745 and the shifted light curve differences. The middle and bottom panels include linear fits with $a$ being the slope of the linear fit.

observed at the NOT (April 1998-November 1999) (see Table 1).

Plots similar to Figs. 1 and 2 are shown in Figs. 3-5. For HE 2149-2745 (Fig. 3) and RX J0911+0551 (Fig. 4) we see some long term variations in the light curve difference but the analysis shows that the slopes differ depending on which of the two light curves are interpolated. In B1600+434 (Fig. 5) one sees clear magnitude variations in the light curve differences but they do not satisfy our second condition for microlensing. By comparison with the top plot we see that they are correlated with the quasar variations (the magnitude bump appears at $\sim 420$ days on all plots).

Thus, while we detect intriguing light curve differences and cannot rule out microlensing variability in these systems, this indicates that the detected magnitude changes may also be attributed to photometric or interpolation errors.

\section{Discussion}

We have demonstrated the existence of a short-term microlensing variability in the photometric data sets of the two quasar systems SBS 1520+530 and FBQ 0951+2635. Interestingly, microlensing has previously been detected spectroscopically and as flux anomalies in both systems (Burud et al. 2002b; Faure et al. 2002; Schechter et al. 1998; Jakobsson et al. 2005). We note that the time scales of the short-term variability detected in the quasars (50-200 days) is similar to the "90 day" events in QSO Q0957+561A,B (see Schild 1996).

In principle one can calculate the masses of the lenses responsible for the microlensing from the time scales of brightness variations $(F W H M)$ and the transverse velocities of the compact objects. An approximate value of the lens mass is given by

$M \approx\left(\frac{\text { time } \times V_{\mathrm{e}}}{R_{\mathrm{E}}}\right)^{2}$.

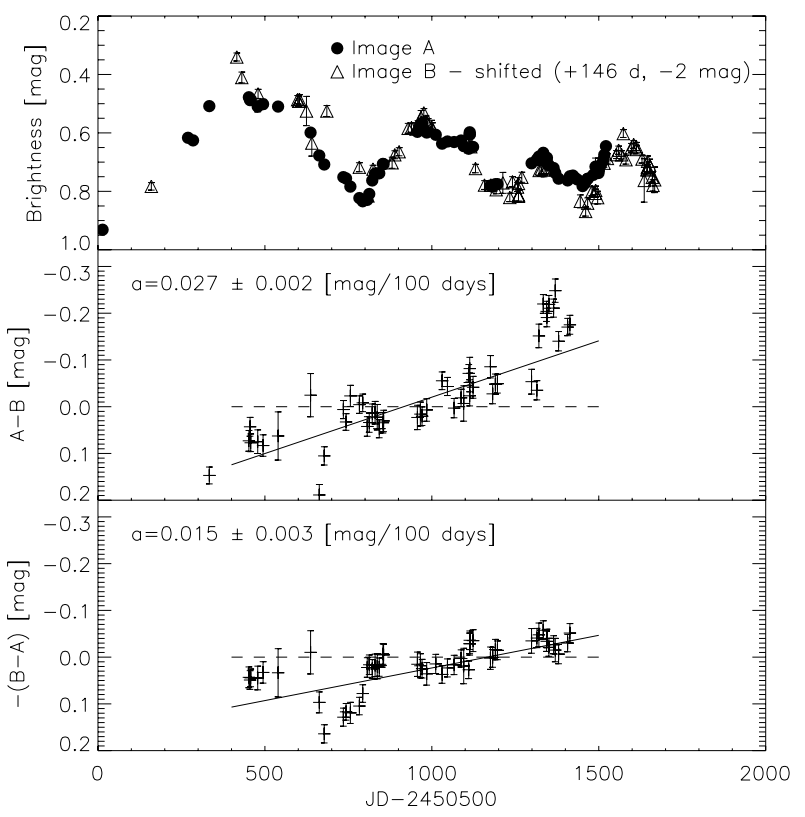

Fig. 4. I-band light curves of RX J0911+055 and the shifted light curve differences. The middle and bottom panels include linear fits with $a$ being the slope of the linear fit.

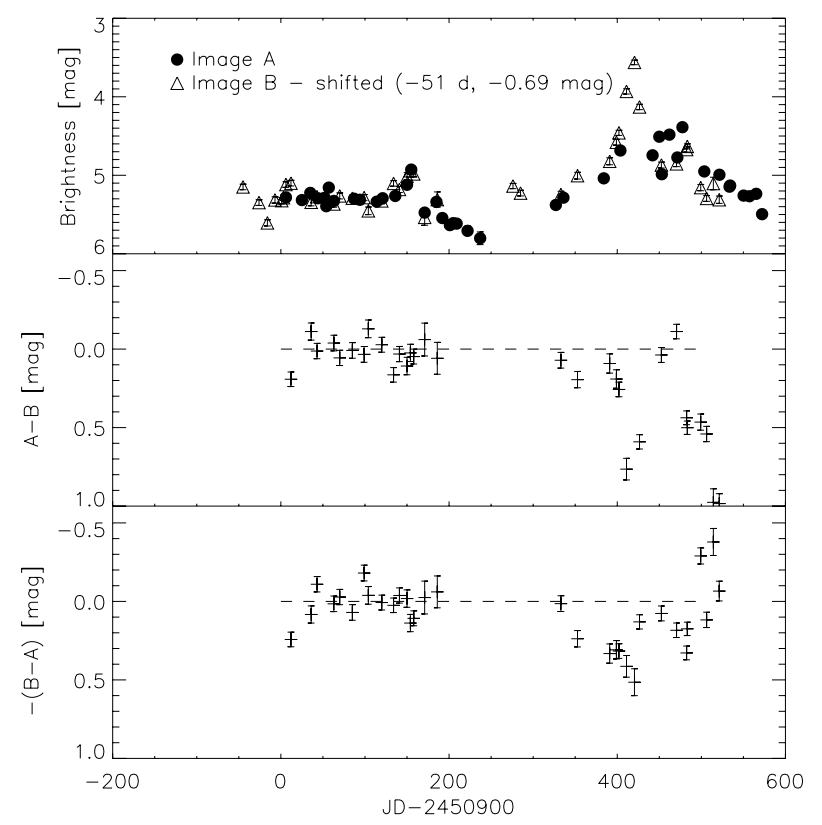

Fig. 5. I-band light curves of B1600+434 and the shifted light curve differences.

Here $V_{\mathrm{e}}$ is an effective source velocity, defined as the change in time of the source position measured by the observer, and $R_{\mathrm{E}}$ is the Einstein radius,

$R_{\mathrm{E}}=\left(\frac{4 G M}{c^{2}} \frac{D_{\mathrm{LS}} D_{\mathrm{S}}}{D_{\mathrm{L}}}\right)^{1 / 2}$,

where $D_{\mathrm{LS}}, D_{\mathrm{S}}$ and $D_{\mathrm{L}}$ are the lens-source, the source-observer and the lens-observer angular diameter distances, respectively ${ }^{1}$.

In Table 2 we show the values of possible microlens masses and the corresponding transverse velocities are given by Eqs. (1)

\footnotetext{
${ }^{1}$ We assume a flat universe with $\Omega_{\mathrm{m}}=0.3, \Omega_{\Lambda}=0.7$ and Hubble constant $H_{0}=70 \mathrm{~km} \mathrm{~s}^{-1} \mathrm{Mpc}^{-1}$.
} 
Table 2. Mass-speed relation for SBS 1520+530 and FBQ 0951+2635.

\begin{tabular}{ccc}
\hline \hline Mass & SBS1520+530 & FBQ 0951+2635 \\
{$\left[M_{\odot}\right]$} & Velocity $\left[\mathrm{km} \mathrm{s}^{-1}\right]$ & Velocity $\left[\mathrm{km} \mathrm{s}^{-1}\right]$ \\
\hline 1.0 & $2.44 \times 10^{4}$ & $6.87 \times 10^{4}$ \\
0.001 & 771 & 2171 \\
0.0001 & 244 & 687 \\
\hline
\end{tabular}

and (2). In these calculations we assume that the microlensing is caused by a single compact object. Even with that simplifying assumption, the lens mass can not be determined without knowing the transverse velocity.

There are two possible limits. Due to short duration of the events the microlenses may actually be nanolenses (planets) with masses of order of $10^{-3} M_{\odot}$ for typical transverse velocities of the source of $200-700 \mathrm{~km} \mathrm{~s}^{-1}$. Similar results were obtained for QSO 0957+561 (Pelt et al. 1998). Conversely, fast microlensing variability can be caused by a solar mass object magnifying small continuum parts of the quasar with relativistic velocities (hot spots) (Schechter et al. 2003) or smooth accretion disk occulted by optically-thick, fast moving clouds (Wyithe \& Loeb 2002). Applying this to the two quasars, for microlens mass $1 M_{\odot}$, we obtain source (hot spot, cloud) transverse velocities of $0.08 c$ for SBS $1520+530$ and $0.23 c$ for FBQ $0951+2635$.

Because of the mass-velocity degeneracy none of these possibilities can be eliminated from a simple analysis. Moreover, we stress that mass determination using Eq. (1) is only possible for single isolated microlensing events and when the transverse velocity is known. More detailed analyses (Lewis et al. 1993; Wyithe \& Turner 2001) assume that the quasar light is affected by numerous compact objects in the foreground galaxy, creating a complex network of caustic lines, and not by a single object (Paczyński 1986).

We conclude that, of the systems we have studied, those with detected flux varability due to microlensing are also reported to have intensity anomalies of spectra continuum and emission line differences. For these systems we have found small linear trends of $\sim 0.005-0.010 \mathrm{mag} / 100$ days and bumps with amplitude of $\sim 0.05 \mathrm{mag}$ and durations of $\sim 100$ days. Interestingly, the systems for which no microlensing variability was detected have no convicing detected microlensing from other methods, although there may be indications in e.g. HE 2149-2745 (Burud et al. 2002a). However, smaller microlensing signals ( $0.005 \mathrm{mag} / 100$ days) in these systems would have been undetectable in our observations.

Acknowledgements. We thank Joachim Wambsganss, Rolf Stabell, Cecile Faure and Jaan Pelt for valuable comments. The Dark Cosmology Centre is funded by the DNRF. This work was carried out within the framework of the EC FP6 Marie Curie Research Training Network "Astrophysics Network for Galaxy LEnsing Studies (ANGLES)". D.P. acknowledges receipt of a research studentship at the Nordic Optical Telescope. The data used are based on observations made with the Nordic Optical Telescope, operated on the island of La Palma jointly by Denmark, Finland, Iceland, Norway, and Sweden, in the Spanish Observatorio del Roque de los Muchachos of the Instituto de Astrofisica de Canarias.

\section{References}

Burud, I., Hjorth, J., Jaunsen, A. O., et al. 2000, ApJ, 544, 117 Burud, I., Courbin, F., Magain, P., et al. 2002a, A\&A, 383, 71 Burud, I., Hjorth, J., Courbin, F., et al. 2002b, A\&A, 391, 481 Chang, K., \& Refsdal, S. 1979, Nature, 282, 561

Faure, C., Courbin, F., Kneib, J. P., et al. 2002, A\&A, 386, 69 Gaynullina, E. R., Schmidt, R. W., Akhunov, T. et al. 2005, A\&A, 440, 53

Hjorth, J., Burud, I., Jaunsen, A. O., et al. 2002, ApJ, 572, L11 Irwin, M. J., Webster, R. L., Hewett, P. C., et al. 1989, AJ, 98, 1989 Jakobsson, P., Hjorth, J., Burud, I., et al. 2005, A\&A, 431, 103 Kochanek, C. S. 2004, ApJ, 605, 58

Lewis, G. F., Miralda-Escudé, J., Richardson, D. C., et al. 1993, MNRAS, 261, 647

Paczyński, B. 1986, ApJ, 301, 503

Pelt, J., Schild, R., Refsdal, S., et al. 1998, A\&A, 336, 829

Schechter, P. L., Gregg, M. D., Becker, R. H., et al. 1998, AJ, 115, 1371

Schechter, P. L., Udalski, A., Szymański, M., et al. 2003, ApJ, 584, 657

Schild, R. E. 1990, AJ, 100, 1771

Schild, R. E. 1996, ApJ, 464, 125

Wyithe, J. S. B., \& Turner, E. L. 2001, MNRAS, 320, 21

Wyithe, J. S. B., \& Loeb, A. 2002, ApJ, 577, 615 